\title{
Discriminability of electrocutaneous stimuli after topical anesthesia: Detection-theory measurement of sensitivity to painful stimuli
}

\author{
R. JOHN IRWIN, MICHAEL J. HAUTUS, NOEL J. DAWSON, \\ DAVID WELCH, and MARK F. BAYLY \\ University of Auckland, Auckland, New Zealand
}

\begin{abstract}
In three experiments on the psychophysical measurement of pain, electrocutaneous currents were applied to the volar surface of the forearm. In the first experiment, a conventional category scaling method was compared with the rating method of signal detection. The results of both methods were analyzed in detection-theory terms to derive receiver operating characteric curves and measures of the discriminability of adjacent currents. The rating method yielded larger discriminability values than the category scale did, and that method was therefore used in the subsequent experiments to examine the effect of a topical anesthetic on discriminability. When the stimuli were applied through surface electrodes, no effect of the topical anesthetic on discriminability was found, but when the stimuli were applied to a more localized area by intradermal needle electrodes, a dose-dependent effect of the anesthetic on discriminability occurred. For this experiment, the slope of the cumulative sensitivity function increased with increasing elapsed time since the removal of the anesthetic. This result is congruent with the theory that discriminability can serve as a measure of sensitivity to painful stimuli.
\end{abstract}

Two major approaches to the psychophysical measurement of pain can be distinguished. One approach requires subjects to estimate in some way the magnitude of their pain: this method involves psychological scaling. A typical implementation of this approach is the Visual Analogue Scale, in which subjects indicate on a line the estimated strength of their pain. The other approach requires subjects to state which of two stimuli is the more painful: this method involves sensory discrimination. A typical implementation of this approach is the detection-theory method of forced choice, in which two experimental stimuli are presented to a subject who must state which is the stronger.

Each of these approaches has its strengths and weaknesses. The Visual Analogue Scale, for example, seems well suited for use in a clinical setting where a detectiontheory method is less obviously applicable. On the other hand, procedures that require subjects to estimate sensory magnitudes suffer from biases that may be impossible to eliminate or to estimate (Poulton, 1989). By contrast, detection-theory methods, while free from biases of that kind, may not reveal anything about the experience of

\footnotetext{
This research was conducted under contract (No. IRW101) between the Foundation for Research, Science and Technology and the first author. We thank Margaret Francis for advice on some of the statistical analyses, and the Astra Division of Pharmaco (New Zealand) Limited for providing the EMLA cream used in the research. Requests for reprints should be sent to R. J. Irwin, Department of Psychology, University of Auckland, Private Bag 92019, Auckland, New Zealand.
}

-Accepted by previous editor, Charles W. Eriksen pain. In an influential article, Rollman (1977) observed that the ability to discriminate between two stimuli may reveal nothing about the painfulness of either. Despite this criticism of detection-theory techniques, they are becoming increasingly recognized as useful tools in the measurement of pain (see, e.g., Goolkasian, 1983; Rollman, Hapidou, \& Jarmain 1990).

There have been many attempts in psychophysics to reconcile these two approaches to the measurement of sensory magnitude, though rarely as they apply to the measurement of pain (Gracely \& Coppola, 1981, have offered one attempt). The objectives of the work reported here were to contribute to that reconciliation by showing how detection-theory methods for measuring pain can provide the same kind of information as more traditional scaling methods, and then to show how the results of those methods are sensitive to the effects of a known anesthetic agent. If discrimination is reduced by such an agent, it is reasonable to conclude that discriminability can serve as an index of sensitivity to painful stimuli.

The psychophysical theory that we employ in an attempt to achieve these objectives has been developed in several places by Laming $(1984,1991)$ and is closely related to the theory presented in a series of papers on hearing by Durlach and Braida and their collaborators (1969; Braida \& Durlach, 1972, and many subsequent papers). The basic idea in this theory is that the magnitudes that a subject ascribes to stimuli can properly be said only to have ordinal properties, not numerical values. As a result, the information available from such judgments is restricted to the way in which different stimuli give rise to different frequencies of responses, and from that information, 
the discriminability of one stimulus from another can be inferred. When examined in this way, estimates of sensory magnitude turn out to provide coarse estimates of sensory discrimination. The estimates are coarse because of the way in which experimental stimuli are arranged in such studies. Typically, a large number of different stimuli are presented in random order and subjects are required to estimate their painfulness. For a wide range of sensory modalities (see Laming, 1984), subjects cannot identify or discriminate between any two of those stimuli with the precision attainable when those two stimuli are presented on their own for discrimination. The degradation in performance is attributable to additional variance entailed in the act of judgment itself. This judgmental variance adds to the sensory or neural variance inherent in the presentation of any stimulus. (The possibility that nonsensory variance may be of consequence in judgments of pain has been raised in a somewhat different way by Coppola \& Gracely, 1983.) The existence of sensory variance, or "noise," is a key concept in the theory of signal detection (Green \& Swets, 1966), a concept that applies with equal force to neural events (see, e.g., Johnson, 1980).

The limits of sensory discrimination can be understood in terms of the statistical decision model of signal detection theory. In the standard version of this model, the stimuli to be discriminated give rise to distributions of sensory effects of different means but common variance. The density functions of the effects are often assumed to be normal, although for some stimuli there may be good reasons for assuming other functions, such as the Rayleigh. On the basis of our previous work with electrocutaneous stimuli (Irwin \& Whitehead, 1991), we adopt the normal model here. The discriminability of two stimuli can then be measured by the index, $d^{\prime}$, which is defined as the distance between the means of the two stimulus distributions in units of their standard deviation.

When results are obtained from several pairs of adjacent stimuli, values of $d^{\prime}$ for adjacent pairs can be cumulated to yield a cumulative sensitivity function whose slope represents the sensitivity of the subject to the stimulus dimension under study. Cumulative sensitivity functions have been reported by Braida and Durlach (1972) for sound intensity, by Macmillan, Kaplan, and Creelman (1977) for speech discrimination, and by Irwin and Whitehead (1991) for electrocutaneous stimuli. An advantage of presenting discriminative performance from several stimuli in this way arises, as Laming (1984) has shown, from the direct comparison that it affords with data derived from experiments on psychological scaling. Through application of the same statistical decision model to scaling experiments, a bias-free interpretation of the results of those experiments can be obtained, and a straightforward comparison can be made of discriminative performance in scaling and discrimination experiments.

A valuable property of the cumulative sensitivity function derives from the fact that it shows the discriminability of several stimuli, not just two. There have been several attempts to show whether an analgesic, for example, affects the discriminability of just two stimuli (e.g., Harkins \& Chapman, 1976), but from the discriminability of two stimuli it may be difficult or impossible to tell, as Rollman (1977) has asserted, whether the sensitivity to both stimuli has been suppressed by the agent and their discriminability has been unaffected, or whether there has been a reduction in discriminability as well. The cumulative sensitivity function makes such an interpretation less ambiguous.

Here we report three experiments in which we used electrocutaneous stimuli. The first experiment was an attempt to replicate some of the work of Irwin and Whitehead (1991). Its purpose was partly to show the robustness of the phenomenon that psychological scaling methods yield reduced measures of discrimination in comparison with standard detection-theory methods, and partly to show the relation between these two procedures in terms of their cumulative sensitivity functions so that it might be seen how conclusions drawn from one function could apply to the other. In the two subsequent experiments, we used a detection-theory method in order to obtain a sensitive measure of discrimination. In both experiments, we measured the discriminability of electrocutaneous stimuli with and without the application of a topical anesthetic to the skin. The reasoning here was that if discriminative performance is related to pain perception, then that performance ought to be degraded by a known anesthetic agent, and the degradation ought to show up as a decrease in slope of the cumulative sensitivity function.

\section{EXPERIMENT 1}

In this experiment, we compared a cumulative sensitivity function derived from a standard detection-theory task, the rating method, with a function derived from a standard psychological scaling task, the category scaling procedure. The same subjects judged the same stimuli (electrocutaneous currents) in both tasks, so that any difference in the obtained sensitivity functions can be attributed to differences in the tasks. The rating task measured the discriminability of a pair of currents; subjects were asked to rate their confidence that the larger of a pair of currents was presented. We call this a discrimination task. In the category task, subjects were asked to assign a label to each current to describe its degree of painfulness. We call this a description task.

\section{Method}

Subjects. Four men (including two of the authors, M.B. and D.W.) took part. They had all signed a consent form approved by the university's Human Subjects Ethics Committee.

Apparatus. A waveform generator produced a 200 -msec train of pulses with a repetition rate of $500 \mathrm{~Hz}$ and a pulse width of $0.2 \mathrm{msec}$. The pulses stimulated an isolated battery-powered unit that acted as a constant current source. The amplitude of the current was controlled by a programmable attenuator that allowed six different currents, separated from each other by $0.5 \mathrm{~dB}$, to be presented. The maximum current had a peak amplitude of $5.1 \mathrm{~mA}$. The current was applied to the volar surface of the nonpreferred 
arm by means of disposable pregelled electrodes $10 \mathrm{~mm}$ in diameter (Nikomed brand), whose centers were separated by $55 \mathrm{~mm}$. The positive electrode was always nearer the wrist. The subject sat in front of a terminal in a booth separated from the controlling apparatus. The screen of the terminal presented instructions for the task, and a keyboard served as the subject's response panel. A set of lights attached to the keyboard informed the subject of the sequence of events in a trial. A computer controlled the experiment, except for a switch on the battery-powered unit which was within reach of the subject and which could be turned off at any time.

Procedure. Each experimental session comprised a series of trials. Every trial began with a warning light followed by a 200 -msec observation interval during which a stimulating current was presented. Each trial ended after the subject entered a response on the keyboard. For the discrimination task, the subjects rated their confidence that the larger of two currents had been presented by entering one of six numbers on the keyboard. For the description task, the subjects judged which one of six categories best described their experience of the stimulus. To do this, they entered a number on the keyboard, from 1 for not painful to 6 for very painful. The two tasks also differed in the sequence in which a stimulus was presented. For the discrimination task, only one of two currents was presented on any trial in an experimental session, but for the description task, one of six currents was presented on any trial of the session. This difference, which typically distinguishes the implementation of these two procedures, is the critical difference that gives rise to the greater variance associated with the description task (see, e.g., Laming, 1984).

In addition to practice and warm-up trials, every pair of stimuli was presented 144 times to each subject in each task over several sessions. The order of undertaking the two tasks was counterbalanced among the 4 subjects, with the different tasks being presented in different sessions.

\section{Results}

The detection theory index of discriminability, $d^{\prime}$, was computed for the five adjacent pairs of currents for each subject for each task. The index was computed by constructing a receiver operating characteristic (ROC) curve for a pair of currents for each subject. The ROC curve is a graph that shows the relation between two conditional probabilities: one is the probability that the subject assigns a particular rating or description, or a more extreme one, given that the larger current has been presented, and the other probability is the probability of that assignment, given the smaller current. In detection theory, these two probabilities have become known as the hit rate and the false-alarm rate.

Forty ROC curves were available for analysis from this experiment (4 subjects $\times 5$ pairs $\times 2$ tasks). The normal-normal equal variance model of detection theory was fitted to each ROC curve by a procedure similar to that outlined by Dorfman and Alf (1969) to provide maximumlikelihood estimates of the discriminability parameter, $d^{\prime}$, and other indices of performance. ${ }^{1}$ In order to provide a summary of the main results, the ROC curves from the 4 subjects were also averaged by means of a jackknifing procedure; this procedure, which involves a resampling plan, enables one to avoid the potential pitfalls of conventional averaging of ROC data (see Dorfman \& Berbaum, 1986). The $d^{\prime}$ values estimated by the jackknife technique are illustrated in the cumulative sensitivity functions in Figure 1. The figure shows a substantial difference in slope of the functions for each task, a result which

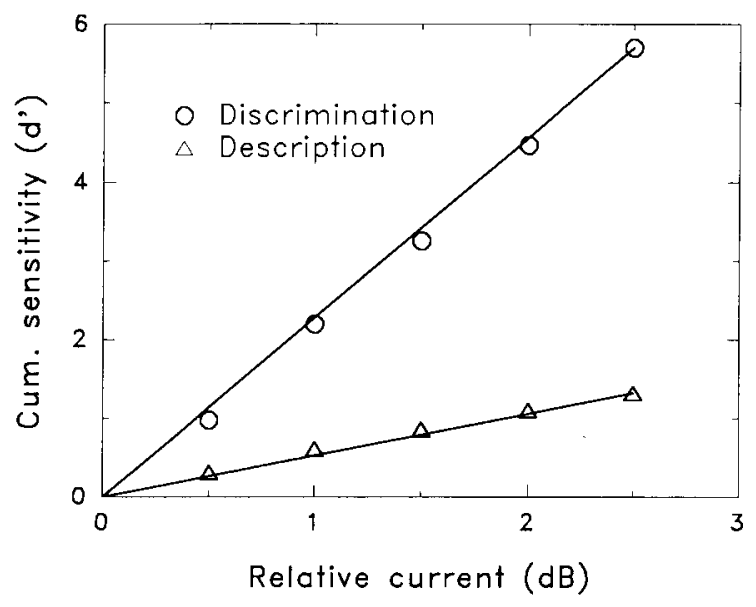

Figure 1. Cumulative sensitivity for the detection-theory discrimination task and the category-scaling description task as a function of relative current. The $d^{\prime}$ values were derived, with the use of jackknife techniques, from the pooled judgments of all subjects. The straight lines are drawn from the origin to the last point of each function.

replicates the work of Irwin and Whitehead (1991). The straight lines drawn between the origin and the last value of each function show the extent to which they conform with Weber's law. The average Weber fraction can be derived from the slope of these lines. Taking a change in $d^{\prime}$ of unity as a just noticeable difference, then the equation $\mathrm{dB}=20 \log [(A+\Delta A) / A]$, where $A$ is the current amplitude, yields the Weber fraction. For the discrimination task, the average Weber fraction for $d^{\prime}=1$ is 0.051 , and for the description task, it is $\mathbf{0 . 2 4 4}$.

\section{Discussion}

The results of this experiment show that categoryscaling judgments of painfulness, when given a bias-free interpretation, provide results similar to those of a discrimination task, but the resolution between stimuli that the category judgments yield is very much reduced in comparison with those yielded by a standard discrimination task. To this extent, our results provide a confirmation of the findings of Irwin and Whitehead (1991) and of Rollman (1979, 1983). A similar result could be expected if this analysis were made of other scaling methods, such as the method of magnitude estimation (Braida \& Durlach, 1972); the category scale is used here merely as an example of scales that require estimations of sensory magnitude. The obtained Weber fraction of 0.051 for the discrimination task is similar to the value of 0.043 reported by Irwin and Whitehead (1991), and it is somewhat smaller than the value of 0.08 obtained by Rollman and Harris (1987), who used the method of limits.

\section{EXPERIMENT 2}

We next examine the ability of the more sensitive discrimination task to detect modulation of the pain system. To this end, we used the rating method to measure the 
effects of a topical anesthetic on the cumulative sensitivity function for the same stimuli as those used in Experiment 1 .

\section{Method}

Subjects. Two of the authors (M.B. and D.W.) served as the subjects for this experiment, which was conducted after Experiment 1 .

Apparatus. The same apparatus as that used in Experiment 1 produced the same set of currents to serve as stimuli.

Procedure. The anesthetic EMLA (an acronym for eutectic mixture of local anesthetics) was a $5 \%$ eutectic mixture of lignocaine and prilocaine in a cream vehicle; $2.5 \mathrm{~g}$ were applied under an occlusive dressing for $1 \mathrm{~h}$ to an area of the ventral forearm approximately $65 \mathrm{~mm}$ long $\times 20 \mathrm{~mm}$ wide. This procedure is clinically effective in making possible, for example, painless venipuncture (Ehrenström-Reiz, Reiz, \& Stockman, 1983).

After a period of $1 \mathrm{~h}$, the EMLA was removed and the electrodes were placed on the skin where the EMLA had previously been. As in Experiment 1, the electrodes were separated by $55 \mathrm{~mm}$. Discrimination was measured by the rating method of signal detection at four time intervals: $15,30,45$, and $60 \mathrm{~min}$ after the EMLA had been removed, and also at those four time intervals without the application of EMLA. The sessions with and without EMLA were alternated and counterbalanced between the 2 subjects. As in Experiment 1,144 trials of each stimulus in a pair were presented.

\section{Results}

Again, the principal tool for analyzing the results of Experiment 2 was the ROC curve. There were 80 ROC curves available from this experiment ( 5 stimulus pairs $\times$ 4 time intervals $\times 2$ subjects $\times 2$ amounts of anesthetic) and the normal-normal equal variance model was fitted to each to obtain maximum-likelihood estimates of the discrimination parameter, $d^{\prime}$. Inspection of these $80 d^{\prime}$ values revealed that the application of the topical anesthetic had little systematic effect on discrimination. This was confirmed by an analysis of variance of the obtained $d^{\prime}$ values.

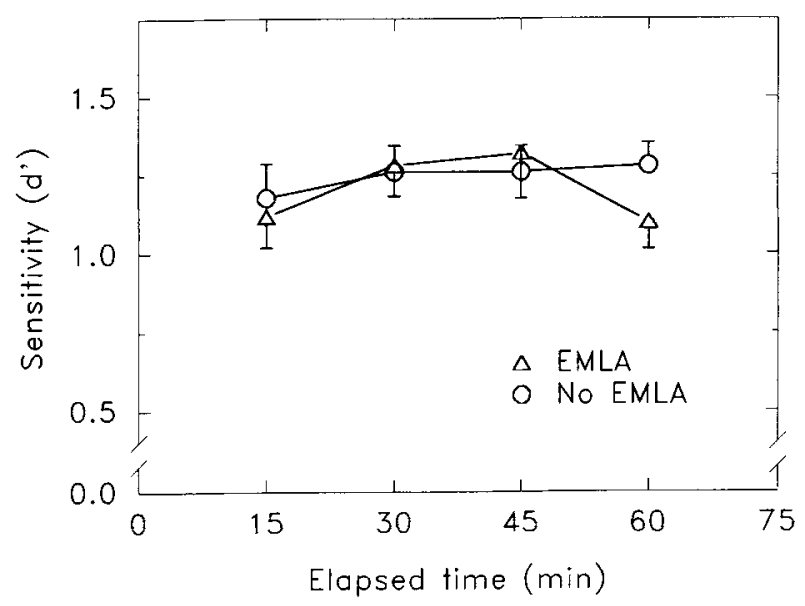

Figure 2. Sensitivity, averaged over subjects as well as all stimulus pairs, obtained in Experiment 2, as a function of elapsed time. The vertical lines represent one standard error about each mean (only one side is shown for clarity).
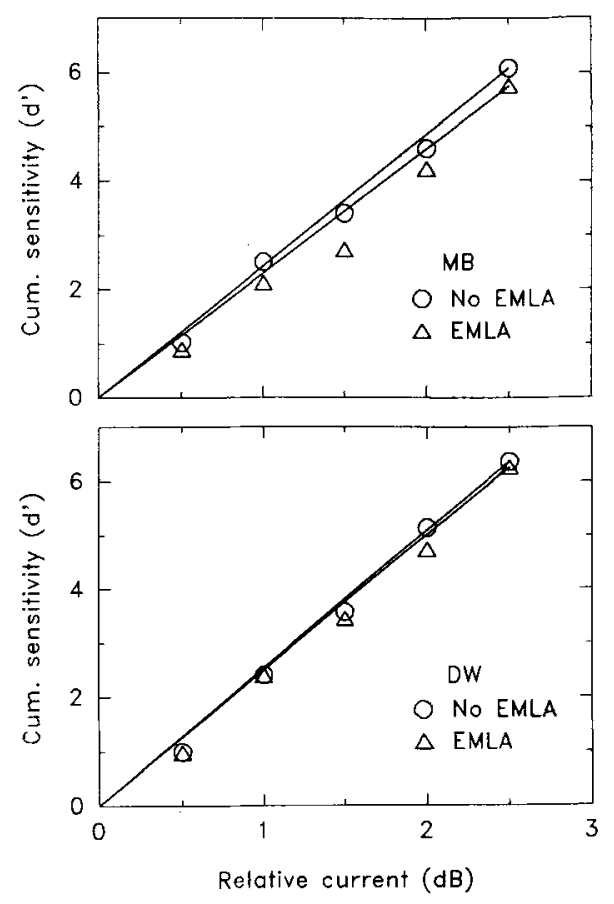

Figure 3. Cumulative sensitivity of each subject as a function of relative current, with and without the application of EMLA, in Experiment 2 . The straight lines are drawn from the origin to the last point of each function.

A comparison of discrimination with and without the application of EMLA yielded $F(1,1)>3.22, p=.324$. The conclusion that the anesthetic had no appreciable effect on the discriminability of the stimuli was strengthened by the finding that there was no significant effect of the time interval since the removal of the anesthetic on discriminability either $[F(3,3)>3.36, p=.173]$. Figure 2 shows discriminability, averaged over both subjects and the five stimulus pairs, as a function of the time elapsed since the removal of EMLA. Clearly no systematic effect of the application of the EMLA or of the time since its administration is discernible.

Because the time since administration of the EMLA had no significant effect on discrimination, the $d^{\prime}$ values from the four time intervals were averaged to produce a summary comparison between the cumulative sensitivity functions obtained with and without topical anesthesia. Figure 3 shows these summary cumulative sensitivity functions for each subject; a small effect of the anesthetic may be discernible in the figure, although the effect is not statistically significant (see above).

\section{Discussion}

A possible reason why the topical anesthetic had no effect on the discriminability of electrocutaneous currents in this experiment is that the discriminability of two electrocutaneous stimuli may be unrelated, as has been often suggested (e.g., by Rollman, 1977), to their painfulness. 
In that case, the anesthetic may have reduced the painfulness of the electrocutaneous stimuli without affecting their discriminability. Another explanation is that the spread of current extended to axons beyond the effective range of the EMLA, at least as it was applied in this experiment. This possibility arises from the fact that the centers of the electrodes used in this experiment were separated by $55 \mathrm{~mm}$, and the active site of an electrode itself was a circular area approximately $10 \mathrm{~mm}$ in diameter.

In order to distinguish between these possibilities, a third experiment was conducted in which an attempt was made to confine the region of electrical stimulation to the likely region of action of the topical anesthetic. In order to accomplish this we used intradermal needles that were inserted just under the skin.

\section{EXPERIMENT 3}

The purpose of this experiment was to study the effect of a topical anesthetic on discrimination of electrocutaneous stimuli when the region of action of the stimuli was confined to an area and depth of stimulation where the anesthetic was most likely to be effective. The main dif- ference between this experiment and Experiment 2, therefore, consisted in the nature of the stimulating electrodes.

\section{Method}

Subjects. Four of the authors, two of whom had taken part in the earlier experiments, served as subjects for this experiment.

Apparatus. Apart from the electrodes, the apparatus was the same as that used in Experiments 1 and 2 . However, because of the different mode of delivery of the current, a new set of stimulus values was chosen. Five current amplitudes, separated from each other by $1 \mathrm{~dB}$, provided four pairs of currents for discrimination with a maximum peak amplitude of $414 \mu \mathrm{A}$. The electrodes were sterile 27-gauge hypodermic needles separated by $2 \mathrm{~mm}$.

Procedure. The needles were inserted intradermally; the insertion was horizontal to the surface of the skin, as superficially as possible, up to the end of the bevelled tip of the needles (approximately $2 \mathrm{~mm}$ ). Discrimination was measured for one pair of currents on any 1 day. The stimulus pairs were presented in a different random order to each subject until the four pairs had been presented on 4 separate days, and then they were presented again on separate days in reverse order for that subject. Each experimental session comprised 100 trials, so that 200 trials were completed for each stimulus pair. Each day began with a "warm-up" block of 100 trials without EMLA, followed, after a 5-min rest, by another 100 trials for the pair under study, again without EMLA. The needles were then removed, and EMLA was applied for $2 \mathrm{~h}$, after which
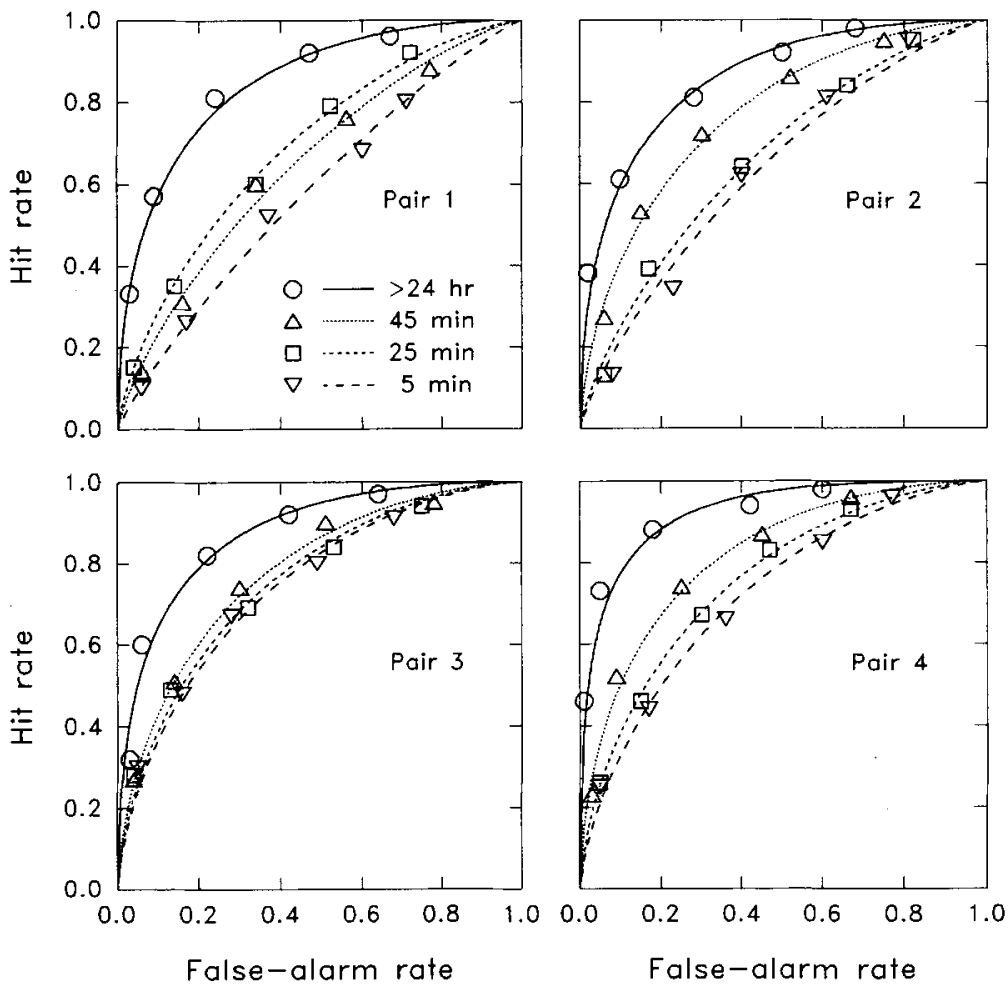

Figure 4. Receiver operating characteristic (ROC) curves fitted with the use of the jackknife technique to the pooled ratings of all subjects in Experiment 3. Each panel shows the ROC curves for a different stimulus pair, and the different symbols show ratings for different elapsed times since the removal of EMLA. 
the needles were reinserted. Then followed three 100 -trial sessions that occurred 5, 25, and $45 \mathrm{~min}$ after the removal of the EMLA. One subject (M.H.) also underwent the procedure in reverse order for two of the stimulus pairs, beginning with the sessions immediately after the removal of EMLA, and finishing with a session $5 \mathrm{~h}$ later after the removal of the EMLA when its effects had presumably completely dissipated.

\section{Results}

Seventy-two ROC curves were available from this experiment: 64 from the main part of the experiment in which the sessions without EMLA preceded those with EMLA, and 8 from the subsidiary part of the experiment in which sessions with EMLA preceded those without. In addition, 16 ROC curves were constructed by jackknifing the individual data from all subjects. These 16 jackknifed ROC curves, which provide a summary of the main results of the experiment, are illustrated in Figure 4. The figure shows how performance depended on the elapsed time since the removal of the topical anesthetic for each stimulus pair. These ROC curves are described reasonably well by the normal-normal equal variance model used throughout this study. It is also clear that the degree of discrimination (represented by the amount that each curve bows toward the top left corner of the square) is always greatest under the absence of the topical anesthetic and declines systematically with temporal proximity to its removal. This result was confirmed by an analysis of variance of the $64 d^{\prime}$ values estimated from the individual ROC curves, for which the effect of elapsed time gave $F(3,9)>7.626, p=.008$. The effect of elapsed time on discriminability of all stimuli is shown in Figure 5, where the reduction in discriminability caused by the effects of the anesthetic can be clearly seen.

Figure 6 shows the cumulative sensitivity functions for the jackknifed values of $d^{\prime}$ for each elapsed time since the removal of the topical anesthetic. As the lines joining the experimental points show, there is a slight upward cur-

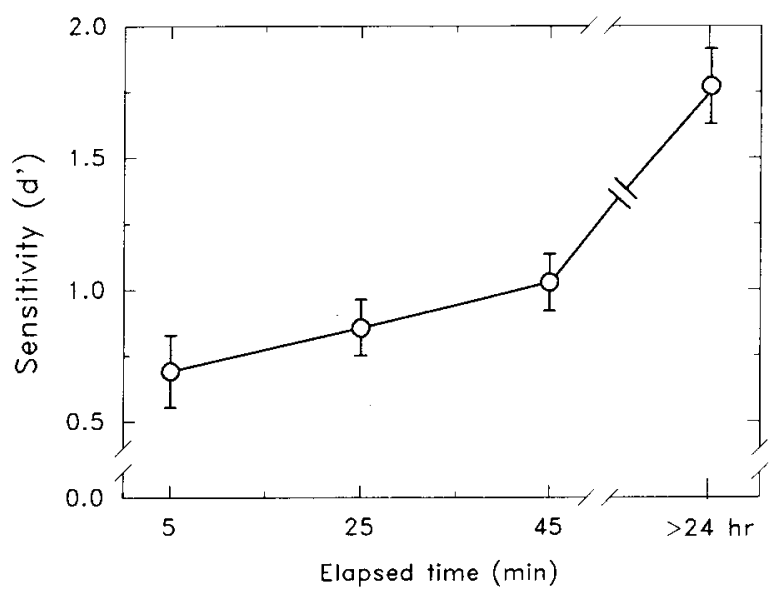

Figure 5. Average sensitivity in Experiment 3 as a function of elapsed time since the removal of EMLA. The vertical lines represent plus and minus one standard error about each mean.

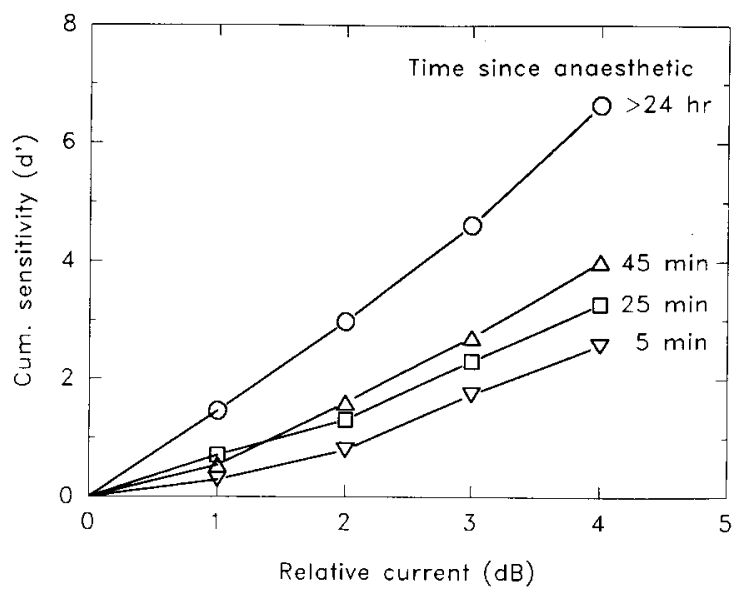

Figure 6. Cumulative sensitivity as a function of relative current, in Experiment 3. The $d^{\prime}$ values were derived, with the use of jackknife techniques, from the pooled judgments of all subjects. The parameter is the elapsed time since the removal of EMLA.

vature in the data, and this nonlinearity represents a departure from Weber's law.

Although the results presented in Figures 4, 5, and 6 show clearly that the topical anesthetic systematically depressed discriminability of pairs of electrocutaneous currents, there was one exception to this average result. Subject N.D. did not show any systematic effect of the application of EMLA on discrimination. This discrepant result may partly stem from an unusual use of the confidence-rating responses. One extreme example for this subject is illustrated by the ROC curve in Figure 7. This curve, obtained $5 \mathrm{~min}$ after the removal of the anesthetic, exhibits a reluctance to state with confidence that the larger stimulus was presented; hence the data points are crowded near the bottom left corner of the square. The path of the curve drawn through those points is not well defined. The estimated value of $d^{\prime}$ for this case is 1.19 , but the standard error of that estimate is 0.24 , which is larger than usual. The $95 \%$ confidence interval for this value of $d^{\prime}$, on the assumption that errors are normally distributed, therefore goes from 0.72 to 1.66 .

For the subject who undertook part of the task in reverse order, with EMLA sessions preceding those without EMLA, the average $d^{\prime}$ values obtained without EMLA were 1.61 and 1.76 for the two orders, and those after the application of EMLA were, on the average, 0.63 and 0.58 for the two orders.

\section{Discussion}

The results of this experiment show that the topical anesthetic EMLA on the average significantly reduced sensitivity to the difference between several pairs of electrocutaneous stimuli (see Figures 4 and 5). Moreover, the reduction in sensitivity was systematically related to the elapsed time since the removal of the anesthetic, and this strengthens the conclusion that discrimination can serve 


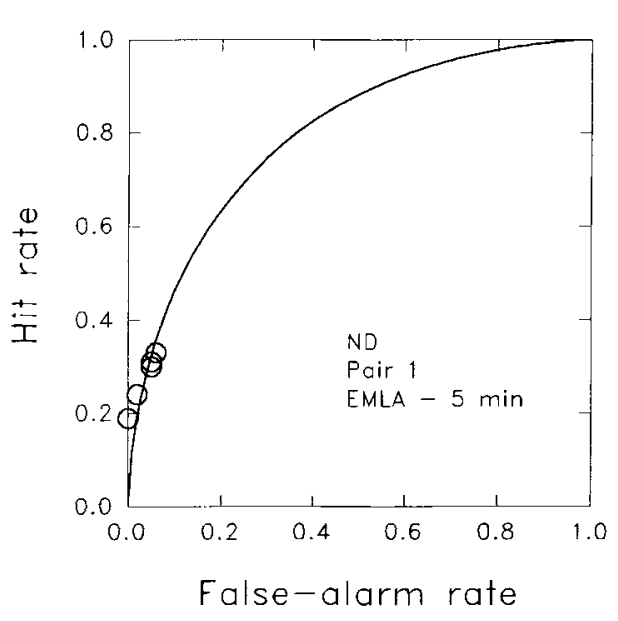

Figure 7. An example of a receiver operating characteristic curve for Subject N.D. in Experiment 3.

as a measure of reduced sensitivity to painful stimulation. On the assumption that the amount of residual anesthetic is inversely related to the time that has elapsed since the removal of the cream, the results in Figures 4, 5, and 6 show a dose-dependent relation between the amount of anesthetic and the degree of suppression of discrimination. It is evident that, in this experiment, in contrast to Experiment 2, we have succeeded in confining the stimulation to a region where the anesthetic acts.

The results of this experiment can be contrasted with those reported by Rollman (1979), who attempted to simulate the effects of an analgesic by reducing the intensity of a stimulating current. He found that the discriminability of a pair of low-intensity stimuli was approximately the same as the discriminability of a pair of high-intensity stimuli. Rollman's experiment and its results, however, are more akin to those from our Experiment 1, where it was also found that the discriminability of currents standing in a constant ratio was approximately constant. Therefore, the effects of an anesthetic such as EMLA cannot be modelled by supposing that the anesthetic provides a uniform attenuation of the stimulating current.

There is a tendency for the data points to curve upward in Figure 7, whereas Weber's law predicts that they would be linear. Weber's law states that the discriminability of two stimuli that stand in a constant ratio is constant. The currents of two stimuli separated by a given decibel difference stand in a constant ratio, so they ought to give rise to equal $d^{\prime}$ values; on the coordinates of Figure 6, Weber's law is therefore a straight line whose slope depends on the Weber fraction. We are not sure why the data depart from Weber's law in this experiment, but it takes the same form as the "near miss" to Weber's law that is occasionally observed for stimulus dimensions (a notable example is the Weber fraction for the amplitude of a sinusoidal tone). In the "near miss," the Weber fraction decreases with increases in stimulus magnitude, and this is the form of the departure from the law in Figure 6.

\section{GENERAL DISCUSSION}

In Experiment 1, a conventional category-scaling technique, in which subjects ascribed labels to their experience of pain, was analyzed in a way similar to the analysis of a bias-free rating method of detection theory. When analyzed in that way, the category method yielded poorer discrimination between any pair of stimuli than the rating method. As a result, the cumulative sensitivity function for the rating method was steeper than that for the category method. The main import of the results of Experiment 1 is that, if the modulation of pain can be assessed by category scaling, as is commonly supposed, then that modulation may be still more readily observable by means of a bias-free discrimination task which, as we have shown, is more sensitive to the dimension under study than the category-scaling procedure.

There was a major difference in the outcomes of $\mathrm{Ex}$ periments 2 and 3: Experiment 2 yielded no effect on discrimination of the topical anesthetic and Experiment 3 resulted in a systematic effect. We believe that the most likely explanation of this difference is to be found in the current spread in the two experiments and in how that current spread was related to the site of action of the anesthetic. Clearly, the shallow penetration and close proximity of the electrode tips in Experiment 3 would have minimized the possibility of current flowing into the deeper tissues of the ventral forearm.

The dose-dependent change in slope of the cumulative sensitivity functions shown in Figure 6 is consistent with the concept that sensitivity to painful stimuli can be measured by their discriminability. The change in slope in Figure 6 takes the form predicted by Irwin and Whitehead (1991) in their analysis of the relation between pain perception and discriminability, and it is consistent with detection-theory conceptions of the process of discrimination. That theory implies that cumulative sensitivity functions should pass through the origin rather than intersect the horizontal axis at some positive value. If, in addition, the functions are linear by virtue of Weber's law, any depression of sensitivity by partial anesthesia will be revealed by a shallower slope of the cumulative sensitivity function.

In the context of the detection-theory analysis offered here, traditional measures of the threshold of pain (e.g., the stimulus which a subject announces is "just painful") can be conceptualized, as Irwin and Whitehead (1991) have done, as a point on the cumulative sensitivity function. That point may depend on linguistic habits and other cultural factors-on an inclination to call a particular stimulus painful-and it will be subject to the biases and context effects of judgments of that kind. The advantage of the detection-theory analysis used here, whether of the more traditional category-scaling method used in Experiment 1 or the discrimination method used in Experiments 2 and 3 , is that the measures that it yields are free of such biases.

We chose electric current as the stimulus for these experiments because of the precision with which it could 
be controlled and delivered. Gracely (1989) has reviewed the advantages and disadvantages of the various stimuli commonly used in the study of experimental pain. A disadvantage of electric current as a stimulus is that it can stimulate a variety of receptors and axons. The receptor population of the skin is complex (Burgess \& Perl, 1973), and the receptors are supplied by both unmyelinated and myelinated axons that vary in diameter and conduction velocity. The susceptibility of these axons to depolarization by electrical stimuli varies inversely with their size. Hence, as the intensity of a stimulus increases, smaller, slower conducting axons are recruited. Thus the perception of the stimulus progresses from something like light touch to noxiousness. Although the stimulus may be unidimensional, its effects are complex. However, this would appear to be a general problem in the study of pain, because other stimuli such as mechanical pressure and heat also have multidimensional effects, ranging from perceptions of touch or warmth to pain.

The work reported here is confined to the study of experimental pain, rather than to acute or chronic clinical pain. Several authors have recognized the contribution that the study of experimental pain can make to understanding clinical pain. For example, Gracely, Lota, Walter, and Dubner (1988) and Gracely (1989) have stated that, in addition to the contribution of basic research on experimental pain to an understanding of the mechanisms underlying pain perception and analgesia, the study of experimental pain can lead to a better understanding of how pain sensations are judged and reported and this understanding can contribute to the assessment of clinical pain. For example, detection theory methods of the kind used here might well be used to assess a patient's response biases, and those biases taken into account in evaluating their reports of clinical pain.

\section{REFERENCES}

BRAIDA, L. D., \& DURLACH, N. I. (1972). Intensity perception: II. Resolution in one-interval paradigms. Journal of the Acoustical Society of America, 51, 483-502.

Burgess, P. R., Perl, E. R. (1973). Cutaneous mechanoreceptors and nociceptors. In A. Iggo (Ed.), Handbook of sensory physiology: Vol. 2. Somatosensory system (pp. 29-78). Berlin: Springer-Verlag.

Coppola, R., \& Gracely, R. H. (1983). Where is the noise in SDT pain assessment? Pain, 17, 257-266.

Dorfman, D. D., AlF, E. (1969). Maximum likelihood estimation of parameters of signal detection theory and determination of confidence intervals-Rating method data. Journal of Mathematical Psychology, 6, 487-496.

Dorfman, D. D., \& Berbaum, K. S. (1986). RSCORE-J: Pooled ratingmethod data: A computer program for analyzing pooled ROC curves. Behavior Research Methods, Instruments, \& Computers, 18, 452-462.

Durlach, N. I., \& BRAIDA, L. D. (1969). Intensity perception: I. Preliminary theory of intensity resolution. Journal of the Acoustical Society of America, 46, 372-383.
Ehrenström-Reiz, G., Reiz, S., \& Stockman, O. (1983). Topical anaesthesia with EMLA, a new lidocaine-prilocaine cream and the cusum technique for detection of minimal application time. Acta Anaesthesiologia Scandinavia, 27, 510-512.

GoOlKasian, P. (1983). An ROC analysis of pain reactions in dysmenorrheic and nondysmenorrheic women. Perception \& Psychophysics, 34, 381-386.

GraCELY, R. H. (1989). Methods of testing pain mechanisms in man. In P. D. Wall \& R. Melzack (Eds.), Textbook on pain (2nd ed., pp. 257-268). Edinburgh: Churchill Livingstone.

Gracely, R. H., \& Coppola, R. (1981). A theory of pain psychophysics: Integrating direct scaling and sensory decision measures. Pain (Suppl. 1), S14.

Gracely, R. H., Lota, L., Walter, D. J., \& Dubner, R. (1988). A multiple random staircase method of psychophysical pain assessment. Pain, 32, 55-63.

GREEN, D. M., \& SwETS, J. A. (1966). Signal detection theory and psychophysics. New York: Wiley.

HaRkins, S. W., \& Chapman, C. R. (1976). Detection and decision factors in pain perception in young and elderly men. Pain, 2, 253-264.

IRWIN, R. J., \& WhITEHEAD, P. R. (1991). Towards an objective psychophysics of pain. Psychological Science, 2, 230-235.

JoHNSON, K. O. (1980). Sensory discrimination: Neural processes preceding discrimination decision. Journal of Neurophysiology, 43, 1793-1815.

LAMING, D. (1984). The relativity of "absolute" judgements. British Journal of Mathematical \& Statistical Psychology, 37, 152-183.

LAMING, D. (1991). Reconciling Fechner and Stevens? Behavioral \& Brain Sciences, 14, 188-191.

Macmillan, N. A., Kaplan, H. L., \& Creelman, C. D. (1977). The psychophysics of categorical perception. Psychological Review, 84, 452-471.

Poulton, E. C. (1989). Bias in quantifying judgments. Hove, U.K.: Erlbaum.

Rollman, G. B. (1977). Signal detection theory measurement of pain: A review and critique. Pain, 3, 187-211.

Rollman, G. B. (1979). Signal detection theory pain measures: Empirical validation studies and adaptation-level effects. Pain, 6, 9-21.

Rollman, G. B. (1983). Multiple subjective representations of experimental pain. In J. J. Bonica, U. Lindblom, \& A. Iggo (Eds.), Advances in pain research and therapy (Vol. 5, pp. 865-869). New York: Raven Press.

Rollman, G. B., Hapidou, E. G., \& Jarmain, S. H. (1990). Gender differences in pain responsiveness: Contributing factors. Pain (Suppl. 5), S314.

Rollman, G. B., \& HaRris, G. (1987). The detectability, discriminability, and perceived magnitude of painful electric shock. Perception \& Psychophysics, 42, 257-268.

\section{NOTE}

1. Of the 192 ROC curves obtained here, 31 were significantly different from the normal-normal equal variance model at the .05 level of significance according to the chi-square goodness-of-fit statistic. When the normal-normal unequal variance model was fitted to these 31 curves, the mean slope parameter (the ratio of the standard deviations of the two distributions) was 1.004 , with a standard deviation of 0.286 . For these cases, therefore, there was not a systematic departure from the equal variance model, for which the slope is unity by definition.

(Manuscript received December 23, 1992; revision accepted for publication July 6,1993 .) 